\title{
Collective modes of Fermi superfluid containing vortices along the BEC-BCS crossover
}

\author{
Tarun Kanti Ghosh and Kazushige Machida \\ Department of Physics, Okayama University, Okayama 700-8530, Japan
}

(Dated: November 14, 2018)

\begin{abstract}
Using the coarse-grain averaged hydrodynamic approach, we calculate all low energy transverse excitation spectrum of a rotating Fermi superfluid containing vortex lattices for all regimes along the BEC-BCS crossover. In the fast rotating regime, the molecular BEC enters into the lowest Landau level, but the superfluid in the unitarity and the BCS regimes occupies many low-lying Landau levels. The difference between the breathing mode frequencies at the BEC and unitarity limit shrinks to zero as the rotation speed approaches the radial trap frequency, in contrast to the finite difference in the non-rotating systems.

PACS numbers: 03.75.Kk,03.75.Ss,03.75.Lm
\end{abstract}

Strongly interacting two-component Fermi gases provide a unique testing ground for the theories of exotic systems in nature. In atomic Fermi gases, tunable strong interactions are produced using Feshbach resonance 1, 2, 3]. By sweeping the magnetic field in the Feshbach resonance experiments, magnitude and nature of the two-body interaction strength changes from repulsive to attractive. Across the resonance the $s$-wave scattering length $a$ goes from large positive to large negative values. The fermionic system becomes molecular Bose-Einstein condensates (BEC) for strong repulsive interaction and transform into the Bardeen-Cooper-Schrieffer (BCS) superfluid when the interaction is attractive. The first observations of BEC of molecules consisting of loosely bound fermionic atoms $4,5,6$ initiated a series of explorations 7, 8, 9, 10, 11, 12 of the crossover between BEC and BCS superfluid. Near the resonance, the zero energy $s$-wave scattering length $a$ exceeds the interparticle spacing and the interparticle interactions are unitarity limited and universal.

One of the hallmarks of superfluidity, be it bosonic or fermionic, is the presence of quantized vortices. Recently, large number of vortices in a Fermi gas along the BEC-BCS crossover are indeed observed [13] and it proofs that this strongly interacting system is really superfluid. Equilibrium properties of vortices in a rotating Fermi superfluid described by BCS theory have already been the subject of several studies [14, 15, 16, 17, 18]. There have been known several outstanding vortex problems associated with the fermionic excitations around a vortex core in the quantum limit of a superconductor under magnetic field [19].

The successful measurements of the breathing mode frequencies in a non-rotating Fermi superfluid [20, 21] and the availability of the vortex lattices in a rotating Fermi superfluid 13. have stimulated us to study theoretically the collective excitation spectrum of a rotating Fermi superfluid containing large number of vortices along the BEC-BCS crossover. So far, both theoretical 22, 23, 24] and experimental [25] results have been obtained for low-energy modes of a rotating, weakly interacting condensates with large number of vortices. As in the case of a rotating bosonic clouds, the frequencies of the collective modes of a Fermi superfluid along the crossover in presence of the vortex lattices can be measured experimentally with high accuracy.

In this work, we calculate all low energy frequencies of the transverse excitations of a strongly interacting Fermi superfluid contains vortex lattices along the BEC-BCS crossover. In particular, we find that the Fermi superfluid in the BEC regime enters into the lowest Landau level at fast rotation. However, the superfluid in the unitarity and the BCS regimes occupy many low-lying Landau levels even at fast rotation. At fast rotation, the radial breathing mode frequency becomes close to $2 \omega_{r}$ ( $\omega_{r}$ is the radial trap frequency) in all the regimes along the crossover, in contrast to the non-monotonous behavior of the breathing mode along the crossover in the non-rotating systems [26].

We consider a large number of vortices formed in a rotating Fermi superfluid confined by a cigar shaped harmonic trap potential $V_{\mathrm{ho}}(r, z)=(M / 2)\left(\omega_{r}^{2} r^{2}+\omega_{z}^{2} z^{2}\right)$. We shall use the concept of diffused vorticity which has been successfully used to study the low energy excitations and the dynamics of the weakly interacting atomic BEC in presence of large number of vortices 22, 23, 24]. The diffused vorticity, $\nabla \times \mathbf{v}=2 \boldsymbol{\Omega}$, of a vortex lattice is an effective coarse-grain averaged description that simplifies the formalism by smoothing out the effect of the individual vortices. The concept of diffused vorticity is sufficient to describe the dynamics at macroscopic distances, larger than the average distance between vortices.

We assume that the system behaves hydrodynamically throughout all the regime. If the system is BCS superfluid, then as long as the oscillation frequency is below the gap frequency $\left(\Delta_{g} / \hbar\right)$ needed to break up a Cooper pair, this condition is expected to be fulfilled. Within the coarse-grain hydrodynamic description, the equations of motion for the density and the velocity field which are written by the following continuity and Euler equations, respectively,

$$
\frac{\partial n}{\partial t}=-\nabla \cdot[n(r) \mathbf{v}]
$$


and

$$
\begin{aligned}
M \frac{\partial \mathbf{v}}{\partial t} & =-\nabla\left[\frac{1}{2} M \mathbf{v}^{2}+V_{\mathrm{ho}}(r, z)-\frac{1}{2} M \Omega^{2} r^{2}+\mu(n)\right] \\
& +2 M \mathbf{v} \times \mathbf{\Omega}+M \mathbf{v} \times \nabla \times \mathbf{v}
\end{aligned}
$$

where $\mu(n)$ is the density-dependent chemical potential. Also, $\mathbf{v}=(\hbar / 2 M) \nabla \theta$ is the superfluid velocity of a particle of mass $M$. The terms $2 M \mathbf{v} \times \Omega$ and $\nabla\left[(1 / 2) M \Omega^{2} r^{2}\right]$ are the Coriolis and the centrifugal forces, respectively. The equation of state enters through the density-dependent chemical potential. We assume the power-law form of the equation of state as $\mu(n)=C n^{\gamma}$. At equilibrium, the density profile takes the form $n_{0}(r)=(\mu / C)^{1 / \gamma}\left(1-\tilde{r}^{2}\right)^{1 / \gamma}$, where $\mu=(M / 2)\left(\omega_{r}^{2}-\Omega^{2}\right) R_{\perp}^{2}$ is the chemical potential, $R_{\perp}$ is the radial size and $\tilde{r}=r / R_{\perp}$. We are interested to study the radial excitations, therefore, we neglect the weak harmonic confinement along the $z$-direction.

We linearize the density, velocity field and the equation of state $\mu(n)$ around their equilibrium values as $n=n_{0}(r)+\delta n, \mathbf{v}=\mathbf{v}_{0}+\delta \mathbf{v}$ and $\mu(n)=\mu\left(n_{0}\right)+$ $\left.(\partial \mu / \partial n)\right|_{n=n_{0}} \delta n$. In the co-rotating frame, the equilibrium velocity field $\mathbf{v}_{0}=0$. Thus we obtain the equations of motion for the density and the velocity fluctuations in the rotating frame as

$$
\frac{\partial \delta n}{\partial t}=-\nabla_{r} \cdot\left[n_{0}(r) \delta \mathbf{v}\right]
$$

and

$$
M \frac{\partial \delta \mathbf{v}}{\partial t}=-\nabla_{r}\left[\left.\frac{\partial \mu(n)}{\partial n}\right|_{n=n_{0}} \delta n\right]+2 M \delta \mathbf{v} \times \boldsymbol{\Omega} .
$$

We shall follow Ref. 24] to determine the relationship between the density and the phase fluctuations in presence of the vortex lattices. We are looking for the normal mode solutions of the density and phase fluctuations as $\delta n \propto e^{i m \phi} e^{-i \omega t}$ and $\delta \theta \propto e^{i m \phi} e^{-i \omega t}$, where $m$ is the azimuthal quantum number, $\phi$ is the polar angle and $\omega$ is the excitation frequency in the rotating frame. Equation (41) can be written in terms of the phase fluctuations after separating into radial and angular components as

$$
i \hbar \omega \frac{\partial \delta \theta}{\partial r}=2 \frac{\partial}{\partial r}\left[\left.\frac{\partial \mu(n)}{\partial n}\right|_{n=n_{0}} \delta n\right]-2 i \hbar \Omega \frac{m}{r} \delta \theta,
$$

and

$$
i \hbar \omega \delta \theta=\left.2 \frac{\partial \mu(n)}{\partial n}\right|_{n=n_{0}} \delta n-2 i \hbar \Omega \frac{r}{m} \frac{\partial \delta \theta}{\partial r} .
$$

Integrating Eq. (5) with respect to $r$, we then get,

$$
i \hbar \omega \delta \theta=\left.2 \frac{\partial \mu(n)}{\partial n}\right|_{n=n_{0}} \delta n-2 i m \hbar \Omega \int d r \frac{1}{r} \delta \theta .
$$

Equating equations (6) and (7), one gets

$$
\int d r \frac{1}{r} \delta \theta-\frac{r}{m^{2}} \frac{\partial \delta \theta}{\partial r}=0 .
$$

Differentiating the above equation on both sides with respect to the radial coordinate $r$ and it gives the Cauchy equation:

$$
\left(r^{2} \frac{\partial^{2}}{\partial r^{2}}+r \frac{\partial}{\partial r}-m^{2}\right) \delta \theta=0
$$

The general solution of the above equation is given by $\delta \theta=c^{+} r^{m}+c^{-} r^{-m}$, where $c^{+}$and $c^{-}$are arbitrary constants. The velocity fluctuation along the radial direction can be obtained as $\delta v_{r}=(\hbar / 2 M)(m / r)\left(c^{+} r^{m}-c^{-} r^{-m}\right)$. The radial velocity can not diverge as $r \rightarrow 0$. Therefore, $c^{-}=0$ for $m>0$ and $c^{+}=0$ for $m<0$. This gives us

$$
\frac{\partial \delta \theta}{\partial r}= \pm \frac{m}{r} \delta \theta
$$

Substituting Eq. (10) into Eq. ([6), we obtain the following relationship between the density and the phase fluctuations:

$$
i(\hbar \omega \pm 2 \hbar \Omega) \delta \theta=\left.2 \frac{\partial \mu(n)}{\partial n}\right|_{n=n_{0}} \delta n .
$$

Taking gradient of Eq. (111) and then substituting it into Eq. (3), we then get,

$$
\begin{aligned}
0 & =\frac{\gamma}{2}\left(1-\tilde{\Omega}^{2}\right) \nabla_{\tilde{r}} \cdot\left[\left(1-\tilde{r}^{2}\right)^{1 / \gamma} \nabla_{\tilde{r}}\left(1-\tilde{r}^{2}\right)^{1-1 / \gamma} \delta n(r)\right] \\
& -\tilde{\omega}_{\alpha}\left(\tilde{\omega}_{\alpha} \pm 2 \tilde{\Omega}\right) \delta n(r),
\end{aligned}
$$

where $\tilde{\omega}=\omega / \omega_{r}$ and $\tilde{\Omega}=\Omega / \omega_{r}$. Here, $\alpha:\left(n_{r}, m\right)$ is a set of two quantum numbers, where $n_{r}$ is the radial quantum number.

When $\Omega=0$, the solutions of Eq. (12) are known analytically [27]. For $\Omega=0$, the eigenspectrum is given by

$$
\tilde{\omega}_{\alpha}^{2}=|m|+2 n_{r}\left[\gamma\left(n_{r}+|m|\right)+1\right],
$$

and the corresponding eigenfunction is given by

$\delta n_{\alpha}(\tilde{r}, \phi) \propto\left(1-\tilde{r}^{2}\right)^{1 / \gamma-1} \tilde{r}^{|m|} P_{n_{r}}^{(1 / \gamma-1,|m|)}\left(2 \tilde{r}^{2}-1\right) e^{i m \phi}$,

where $P_{n}^{(a, b)}(x)$ is a Jacobi polynomial of order $n$. Assuming the net effect of a coarse grain averaged vortex lattice on the wave functions to be a modification of the effective trapping potential. Therefore, we shall use the above wave function as our ansatz. With this ansatz for $\delta n(\tilde{r}, \phi)$, the expectation value of Eq. (12) is given by

$$
\tilde{\omega}_{\alpha}\left(\tilde{\omega}_{\alpha} \pm 2 \tilde{\Omega}\right)=\left[|m|+2 n_{r} \gamma\left(n_{r}+|m|+1 / \gamma\right)\right]\left(1-\tilde{\Omega}^{2}\right) .
$$

The above quadratic equation can be solved to get the following eigenfrequencies in the rotating frame:

$$
\tilde{\omega}_{\alpha}=\sqrt{\left[|m|+2 n_{r} \gamma\left(n_{r}+|m|+1 / \gamma\right)\right]\left(1-\tilde{\Omega}^{2}\right)+\tilde{\Omega}^{2}} \mp \tilde{\Omega} .
$$

The eigen frequencies in the laboratory frame are obtained by using the simple relation $\omega_{\text {rot }}=\omega_{\text {lab }}-m \Omega$ and it is given by

$$
\begin{aligned}
\tilde{\omega}_{\alpha} & =\sqrt{\left[|m|+2 n_{r} \gamma\left(n_{r}+|m|+1 / \gamma\right)\right]\left(1-\tilde{\Omega}^{2}\right)+\tilde{\Omega}^{2}} \\
& \pm(|m|-1) \tilde{\Omega} .
\end{aligned}
$$


The frequencies of the $m=0$ modes are identical in both the rotating and the laboratory frames and the energy degeneracy is restored for the $m= \pm 1$ modes in the laboratory frame. The dipole mode gives $\tilde{\omega}_{0, \pm 1}=1$ for all $\Omega$ which satisfies the generalized Kohn's theorem.

When $\gamma=1$, it reproduces the known result for weakly interacting rotating BEC with a large number of vortices [24]. The eigenfrequencies given in Eq. (17) with $\gamma=1$ successfully describes the low energy transverse excitations of a rotating atomic BEC containing large number of vortices 24]. In particular, the first $\left(\omega_{1,0}\right)$ and second $\left(\omega_{2,0}\right)$ radial breathing modes are in good agreement with the experimental as well as numerical results of weakly interacting BEC [25, 28]. Moreover, the quadrupole modes $\left(\omega_{0, \pm 2}\right)$ are in good agreement with the numerical results obtained by solving the Bogoliubov-de Gennes equation 29.

At zero temperature, the energy per particle of a dilute Fermi system can be written as $\epsilon=(3 / 5) E_{F} \epsilon(y)$, where $E_{F}=\hbar^{2} k_{F}^{2} / 2 M$ is the free particle Fermi energy and $\epsilon(y)$ is a function of the interaction parameter $y=1 / k_{F} a$. The Monte Carlo calculations of Refs. 30, 31] find $\epsilon(y \rightarrow$ $0)=0.42 \pm 0.01$ which is in fair agreement with the experiments [9, 10]. On the basis of the data of Ref. [31], Manini and Salasnich [32] proposed the following analytical fitting formula of $\epsilon(y)$ for all the regimes along the BEC-BCS crossover:

$$
\epsilon(y)=\alpha_{1}-\alpha_{2} \tan ^{-1}\left[\alpha_{3} y \frac{\beta_{1}+|y|}{\beta_{2}+|y|}\right]
$$

This analytical expression is well fitted with the data of Ref. 31] for a wide range of $y$ on both sides of the resonance. Two-different sets of parameters are considered in Ref. 32]: one set in the BCS regime $(y<0)$ and another set in the BEC regime $(y>0)$. In the BCS limit, the values of the parameters 32 are $\alpha_{1}=0.42, \alpha_{2}=0.3692$, $\alpha_{3}=1.044, \beta_{1}=1.4328$ and $\beta_{2}=0.5523$. In the BEC limit, the values of the parameters [32] are $\alpha_{1}=0.42$, $\alpha_{2}=0.2674, \alpha_{3}=5.04, \beta_{1}=0.1126$ and $\beta_{2}=0.4552$.

The chemical potential $\mu$ is given by 32 ]

$$
\mu=\epsilon(n)+n \frac{d \epsilon(n)}{d n}=E_{F}\left[\epsilon(y)-\frac{y}{5} \epsilon^{\prime}(y)\right]=E_{F} F(y),
$$

where $\epsilon^{\prime}(y)=\partial \epsilon(y) / \partial y$ and $F(y)=\epsilon(y)-(y / 5) \epsilon^{\prime}(y)$. Due to the effect of the solid body rotation and the harmonic trapping potential, the free Fermi energy can be written as $E_{F}=\hbar \omega_{r}\left[3 N \lambda\left(1-\tilde{\Omega}^{2}\right)\right]^{1 / 3}$ with $\lambda=\omega_{z} / \omega_{r}$. The radial and the axial sizes can be written, respectively, as $R_{\perp}=a_{r}(24 N \lambda)^{1 / 6}\left(1-\tilde{\Omega}^{2}\right)^{-1 / 3} \sqrt{F(y)}$ and $R_{z}=$ $a_{z}\left(24 N / \lambda^{2}\right)^{1 / 6}\left(1-\tilde{\Omega}^{2}\right)^{1 / 6} \sqrt{F(y)}$, where $a_{i}=\sqrt{\hbar / M \omega_{i}}$ is the harmonic oscillator length with $i=r, z$. The aspect ratio is given by $R_{z} / R_{\perp}=\left(1-\tilde{\Omega}^{2}\right)^{1 / 2} / \lambda$ which shows that the rapidly rotating Fermi superfluid takes a pancake form even if the confining trap has a cigar shape. It is interesting to note that the aspect ratio is the same for all the regimes along the BEC-BCS crossover.

It is seen from Eq. (17) that when $\Omega \rightarrow \omega_{r}$ all transverse modes with $m=0$ in all the regimes along the

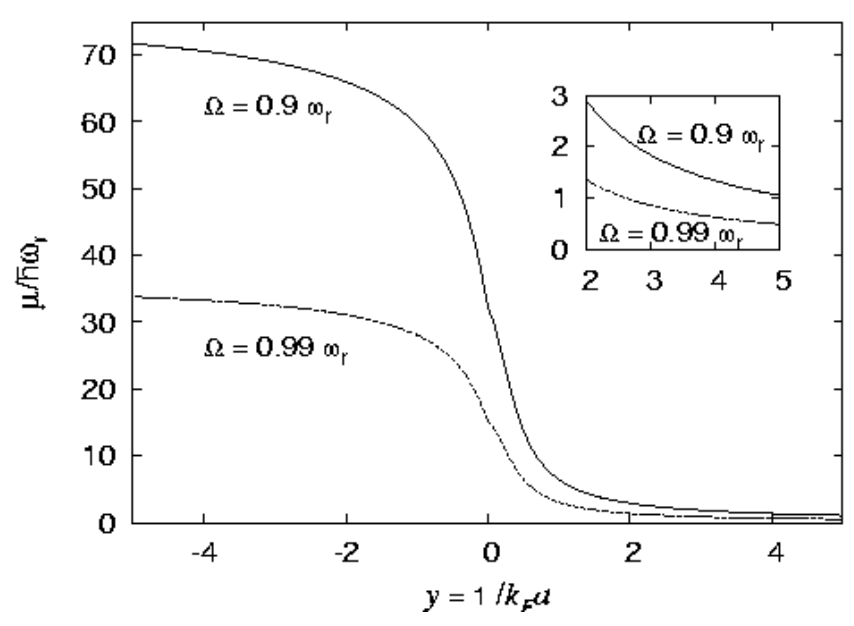

FIG. 1: Plots of the chemical potential vs coupling parameter $y$ of a trapped Fermi gas with $N=2 \times 10^{6}$ and $\lambda=23 / 57$.

BEC-BCS crossover approach to the degenerate value $2 \omega_{r}$. Such a macroscopic degeneracy is reminiscent of the degeneracy of the energy levels of a single particle in an uniform magnetic field, leading to the well known Landau level structure. However, we should mention that our results here hold not for a single particle, but for a system of interacting particles along the BEC-BCS crossover. Therefore, the strongly interacting, rotating Fermi superfluid forms the Landau level structure when $\Omega \rightarrow \omega_{r}$. In Fig. 1, we plot the chemical potential for various values of $\Omega$. Fig. 1 shows that the chemical potential in the BEC side is less than the cyclotron energy gap $\left(2 \hbar \omega_{r}\right)$ at the fast rotation and the system enters into the lowest Landau level. However, at the fast rotation, the chemical potential in the unitarity and in the BCS regimes are quite large compared to the cyclotron energy gap. The fermionic superfluid in the BCS as well as in the unitarity regimes occupies many low-lying Landau levels even at $\Omega \rightarrow \omega_{r}$.

One can extract an effective adiabatic index $\gamma$ and its dependence on the scattering length $a$ by defining the logarithmic derivative as 32

$$
\gamma \equiv \bar{\gamma}=\frac{n}{\mu} \frac{d \mu}{d n}=\frac{\frac{2}{3}-\frac{2 y}{5} \epsilon^{\prime}(y)+\frac{y^{2}}{15} \epsilon^{\prime \prime}(y)}{\epsilon(y)-\frac{y}{5} \epsilon^{\prime}(y)}
$$

From Eq. (17), the first radial breathing mode frequency can be written as

$$
\tilde{\omega}_{1}=\sqrt{2(\gamma+1)\left(1-\tilde{\Omega}^{2}\right)+\tilde{\Omega}^{2}}+\tilde{\Omega} .
$$

When $\Omega=0$, the difference between the breathing mode frequencies at the BEC limit and unitarity limit is $\Delta(\Omega=0)=\omega_{1}(\gamma=1)-\omega_{1}(\gamma=2 / 3)=(2-$ $\sqrt{10 / 3}) \omega_{r}=0.174258 \omega_{r}$. Recently, the radial breathing mode frequency of a non-rotating Fermi superfluid along the crossover has been measured [20, 21] which is 


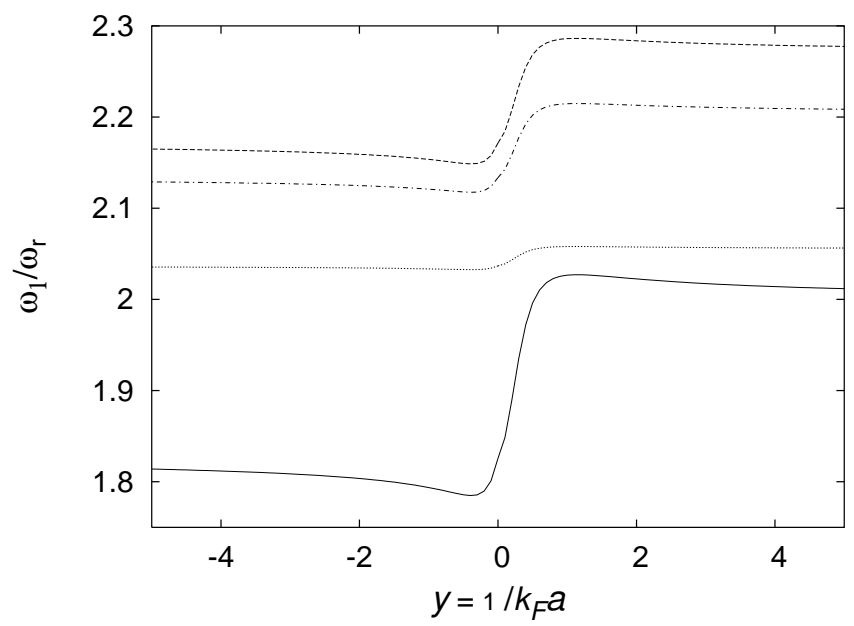

FIG. 2: Plots of the first breathing mode frequency for $\tilde{\Omega}=0$ (solid line), $\tilde{\Omega}=0.75$ (dashed line), $\tilde{\Omega}=0.85$ (dot-dashed line) and $\tilde{\Omega}=0.97$ (dotted line).

in fair agreement with the theoretical result $\omega_{1}(\Omega=0)=$ $\sqrt{2(\gamma+1)} \omega_{r}$. In Fig. 2, we plot the first radial breathing mode in all the regimes along the BEC-BCS crossover for various values of $\Omega$. It is interesting to see from Fig. 2 that the difference between the breathing mode frequencies at the BEC-limit and the unitarity limit decreases as $\Omega$ increases. In fact, the breathing mode in all the regimes approaches $2 \omega_{r}$ when $\Omega \rightarrow \omega_{r}$. The shrinking of the difference of the breathing mode frequency could be observed in the future experiment.

In conclusion, we have obtained an analytic expression for the collective excitation spectrum of a Fermi superfluid containing vortices along the BEC-BCS crossover. The spectrum reduces to the Landau level structure in the fast rotating regime. The superfluid in the BEC regime goes into the lowest Landau level when $\Omega \rightarrow \omega_{r}$. However, the superfluid in the unitarity and the BCS regimes occupies many low-lying Landau levels at the fast rotating regime. The difference between the breathing mode frequencies at the unitarity and the BEC limits shrinks to zero in the fast rotating regime.

\section{Acknowledgments}

This work of TKG was supported by a grant (Grant No. P04311) of the Japan Society for the Promotion of Science.
[1] M. Houbiers, H. T. C. Stoof, W. I. McAlexander, and R. G. Hulet, Phys. Rev. A 57, 1497(R) (1998).

[2] W. C. Stwalley, Phys. Rev. Lett. 37, 1628 (1976).

[3] E. Tiesinga, B. J. Verhaar, and H. T. C. Stoof, Phys. Rev. A 47, 4114 (1993).

[4] M. Greiner, C. A. Regal, and D. S. Jin, Nature 426, 537 (2003).

[5] S. Jochim, M. Bartenstein, A. Altmeyer, G. Hendl, S. Riedl, C. Chin, J. Hecker Denschlag, and R. Grimm, Science 302, 2101 (2003).

[6] M. W. Zwierlein, C. A. Stan, C. H. Schunck, S. M. F. Raupach, S. Gupta, Z. Hadzibabic, and W. Ketterle, Phys. Rev. Lett. 91, 250401 (1993).

[7] K. M. O'Hara, S. L. Hemmer, M. E. Gehm, S. R. Grande, and J. E. Thomas, Science 298, 217 (2002).

[8] C. A. Regal, M. Greiner, and D. S. Jin, Phys. Rev. Lett. 92, 040403 (2004).

[9] T. Bourdel, J. Cubizolles, L. Khaykovich, K. M. F. Magalhae, S. J. J. M. F. Kokkelmans, G. V. Shlyapnikov, and C. Salomon, Phys. Rev. Lett. 91, 020402 (2003).

[10] M. Bartenstein, A. Altmeyer, S. Riedl, S. Jochim, C. Chin, J. H. Denschlag, and R. Grimm, Phys. Rev. Lett. 92, 120401 (2004).

[11] T. Bourdel, L. Khaykovich, J. Cubizolles, J. Zhang, F. Chevy, M. Teichmann, L. Tarruell, S. J. J. M. F. Kokkelmans, and C. Salomon, Phys. Rev. Lett. 93, 050401 (2004).

[12] C. Chin, M. Bartenstein, A. Altmeyer, S. Riedl, S. Jochim, J. H. Denschlag, and R. Grimm, Science 305, 1128 (2004).

[13] M. W. Zweirlein, J. R. Abo-Shaeer, A. Schirotzek, C. H.
Schunck, and W. Ketterle, Nature 435, 1047 (2005).

[14] D. L. Feder, Phys. Rev. Lett. 93, 200406 (2004).

[15] N. Nygaard, G. M. Bruun, C. W. Clark, and D. L. Feder, Phys. Rev. Lett. 90, 210402 (2003).

[16] A. Bulgac and Y. Yu, Phys. Rev. Lett. 91, 190404 (2003).

[17] J. Tempere, M. Wouters, and J. T. Devreese, Phys. Rev. A 71, 033631 (2005).

[18] G. Tonini and Y. Castin, cond-mat/0504612

[19] N. Hayashi, T. Isoshima, M. Ichioka, and K. Machida, Phys. Rev. Lett. 80, 2921 (1998).

[20] J. Kinast, S. L. Hemmer, M. E. Gehm, A. Turlapov, and J. E. Thomas, Phys. Rev. Lett. 92, 150402 (2004).

[21] M. Bartenstein, A. Altmeyer, S. Riedl, S. Jochim, C. Chin, J. H. Denschlag, and R. Grimm, Phys. Rev. Lett. 92, 203201 (2004).

[22] M. Cozzini and S. Stringari, Phys. Rev. A 67, 041602(R) (2003).

[23] F. Chevy and S. Stringari, Phys. Rev. A 68, 053601 (2003).

[24] S. Choi, L. O. Baksmaty, S. J. Woo, and N. P. Bigelow, Phys. Rev. A 68, 031605(R) (2003).

[25] I. Coddington, P. Engels, V. Schweikhard, and E. A. Cornell, Phys. Rev. Lett. 91, 100402 (2003).

[26] S. Stringari, Europhys. Lett. 65, 749 (2004).

[27] T. K. Ghosh and K. Machida, to appear in Phys. Rev. A. Preprint: cond-mat/0510160.

[28] L. O. Baskmaty, S. J. Woo, S. Choi, and N. P. Bigelow, Phys. Rev. Lett. 92, 160405 (2004).

[29] T. Mizushima, Ph. D. Thesis, Okayama University, Okayama, Japan.

[30] J. Carlson, S.-Y. Chang, V. R. Pandharipande, and K. 
E. Schmidt, Phys. Rev. Lett. 91, 050401 (2003).

[31] G. E. Astrakharchik, J. Boronat, J. Casulleras, and S. Giorgini, Phys. Rev. Lett. 93, 200404 (2004).
[32] N. Manini and L. Salasnich, Phys. Rev. A 71, 033625 (2005). 